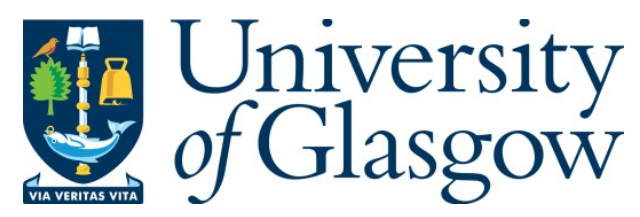

Khan, J. S., Tahir, A., Ahmad, J., Shah, S. A., Abbasi, Q. H., Russell, G. and Buchanan, W. (2020) 5G-FOG: Freezing of Gait Identification in Multi-Class Softmax Neural Network Exploiting 5G Spectrum. In: Computing Conference 2020, London, UK, 16-17 Jul 2020, pp. 26-36. ISBN 9783030522421

(doi:10.1007/978-3-030-52243-8 3)

This is the Author Accepted Manuscript.

There may be differences between this version and the published version. You are advised to consult the publisher's version if you wish to cite from it.

https://eprints.gla.ac.uk/204487/

Deposited on: 2 December 2019

Enlighten - Research publications by members of the University of Glasgow http://eprints.gla.ac.uk 


\title{
5G-FOG: Freezing of Gait Identification in Multi-Class Softmax Neural Network Exploiting 5G Spectrum
}

\author{
Jan Sher Khan ${ }^{1}$, Ahsen Tahir ${ }^{2}$, Jawad Ahmad ${ }^{3}$, Syed Aziz Shah ${ }^{4}$, \\ Qammer H. Abbasi ${ }^{5}$, Gordon Russell ${ }^{3}$, and William Buchanan ${ }^{3}$ \\ 1 University of Gaziantep, Gaziantep, Turkey \\ 2 Glasgow Caledonain University, Glasgow, UK \\ 3 Edinburgh Napier University, Edinburgh, UK \\ 4 Manchester Metropolitan University, Manchester, UK \\ ${ }^{5}$ University of Glasgow, Glasgow, UK
}

\begin{abstract}
Freezing of gait (FOG) is one of the most incapacitating and disconcerting symptom in Parkinson's disease (PD). FOG is the result of neural control disorder and motor impairments, which severely impedes forward locomotion. This paper presents the exploitation of $5 \mathrm{G}$ spectrum operating at $4.8 \mathrm{GHz}$ (a potential Chinese frequency band for Internet of Things) to detect the freezing episodes experienced by PD patients. The core idea is to utilize wireless devices such as network interface card, $\mathrm{RF}$ signal generator and dipole antennas to extract the wireless channel characteristics containing the variances amplitude information that can be integrated into the $5 \mathrm{G}$ communication system. Five different human activities were performed including sitting on chair, slow-walk, fast-walk, voluntary stop and FOG episodes. A multi-class, multilayer full softmax neural network was trained on the obtained data for classification and performance evaluation of the proposed system. A high classification accuracy of $99.3 \%$ was achieved for the aforementioned activities, compared with the existing state-of-the-art detection systems.
\end{abstract}

Keywords: Parkinson's disease, FOG, Classification, Softmax neural network

\section{Introduction}

Parkinson's disease (PD) is a progressive neurodegenerative disease described by Parkinson in 1817 [1]. Over time, PD effectively progresses and worsen and hence called a progressive disease. A specific type of neuron known as dopamine neuron losses during PD that causes FOG. FOG is a serious gait disorder which interrupts walking with a transient and sudden nature. Due to sudden and serious debilitating nature, FOG disturbs the balance of PD patients and therefore causes falls that may lead to mortality $[2,3]$. The pathophysiology of FOG is still under research and its treatment is a still an open clinical challenge [4]. However, recently, authors in [5] reported the impact of Levodopa-Carbidopa 
Intestinal Gel (LCIG) FOG and concluded that a long term control over FOG is possible via LCIG if FOG is detected correctly. Furthermore, it is suggested in [5] that a number of experiments are required with correct identification of FOG in patients.

Authors in [6] reduced FOG and improved mobility via simultaneously targeting motor and cognitive regions through transcranial direct current stimulation. Though, authors [6] reported the improvement of mobility but correctly predicting the state of freezing was overlooked. Therefore, to decrease the fall rate and before providing a solution for FOG, a system must be developed to detect FOG with higher accuracy. FOG can detected through numerous detection systems such as wearable devices and camera etc [7-11]. However, there are several limitations associated with camera-based and wearable based systems. For instance, the camera-based system works raise privacy concerns due to the constant recording of images or videos. In addition, they are computationally expensive as well since processing images or videos require dedicated hardware. On the other hand, wearable devices have to be worn by the subject's all the time due to which the patients might feel uncomfortable. Moreover, more often than not, the patients forget to wear the devices after changing clothes or taking a shower. Due to aforementioned issues, it is evident that other digital medium should be investigated. This paper presents a wireless channel information (WCI) based new detection method. A device free wireless sensing method is developed and the accuracy of the proposed scheme is tested using artificial neural network (ANN).

Over the past few years, ANN has been applied in a number of areas including speech recognition [12], image classification [13], and energy demand prediction. Rahim et al. [12] and Chu et al. [14] applied ANN to the speech recognition. Moreover, ANN-based algorithms have also been used in image classification and recognition $[13,15,16]$. Previously, Neural network based schemes are applied to chemical-related research, molecular biology, medicines, environmental sciences and ecosystems [17-20]. This paper exploits the application of multi-class, full softmax multilayer feedforward neural network (ML-FFNN) using WCI and 5G spectrum for FOG detection.

The core idea of the proposed work is to detect the FOG episode by classifying various human activities such as sitting/standing on chair, slow-walk, fast-walk, voluntary stop. The classification performed using variations in WCI data is received through wireless devices including RF signal generator, networks interface card (NIC) and dipole antenna [21-24].

\section{Experimental Setup}

The general experimental setup for FOG detection is shown in Fig. 1. The experiment was conducted in a room with dimensions (15 meters $\times 15$ meters) in New Science Building, Xidian University, China. The experimental settings included 
an RF generator (DSG3000 Series), two dipole antennas, TP-link (PCE-AC68) next generation dual-band wireless AC1900 PCIe adapter NIC, and HP desktop computer with Ubuntu 14.10 (64 bits) and 4 GB RAM. The RF signal generator connected with the dipole antenna operating at $4.8 \mathrm{GHz}$ was set as an Access Point (AP) to generate RF signals at multiple frequencies. The network interface card wired with dipole antenna embedded in a desktop computer received the seamless WCI data. The transmitter and receiver were kept 10 meters apart from each other.

A total number of 15 volunteers took part in the experimental campaign and were asked to perform the aforementioned five activities. Each human activity constantly disturbed the wireless medium and the unique WCI imprint induced was used for activity recognition.

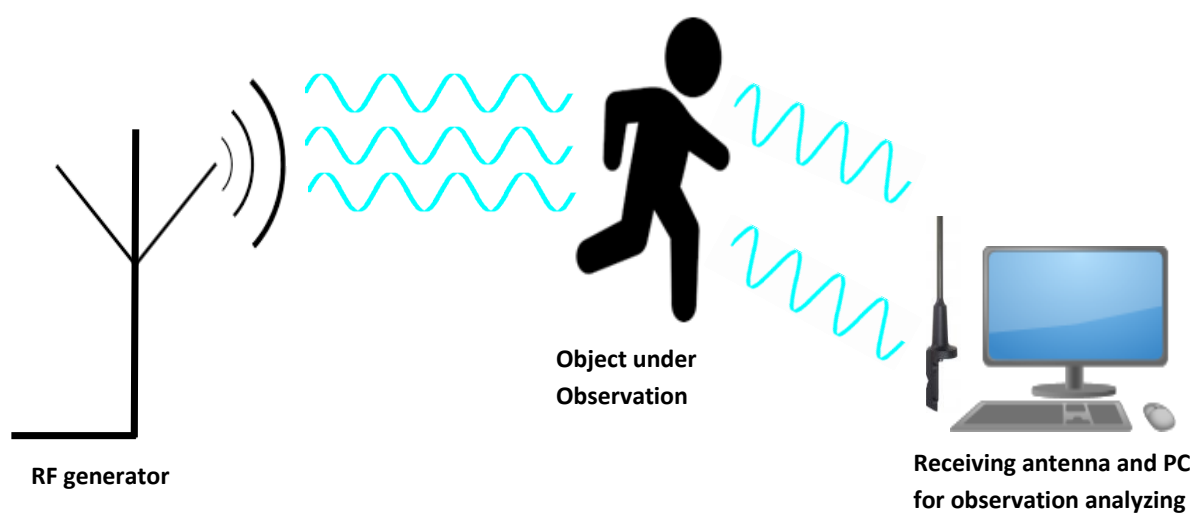

Fig. 1: General setup of the experiment.

\section{FOG Detection Methodology}

The design of the FOG system is presented in Fig. 2 which consists of three main parts:

1). Wireless channel information

2). Feature extraction

3). Multi-class softmax ML-FFNN training \& classification for FOG detection

Step 1: Exploiting the IEEE 802.11n standards for orthogonal frequency division multiplexing (OFDM), which divide a single channel carrier into several subcarriers and enables the data to be transmitted in parallel to solve multipath fading problem [25]. The signal received using network interface card can be computed as:

$$
\boldsymbol{Y}=(\boldsymbol{H} \times \boldsymbol{X})+\boldsymbol{N}
$$




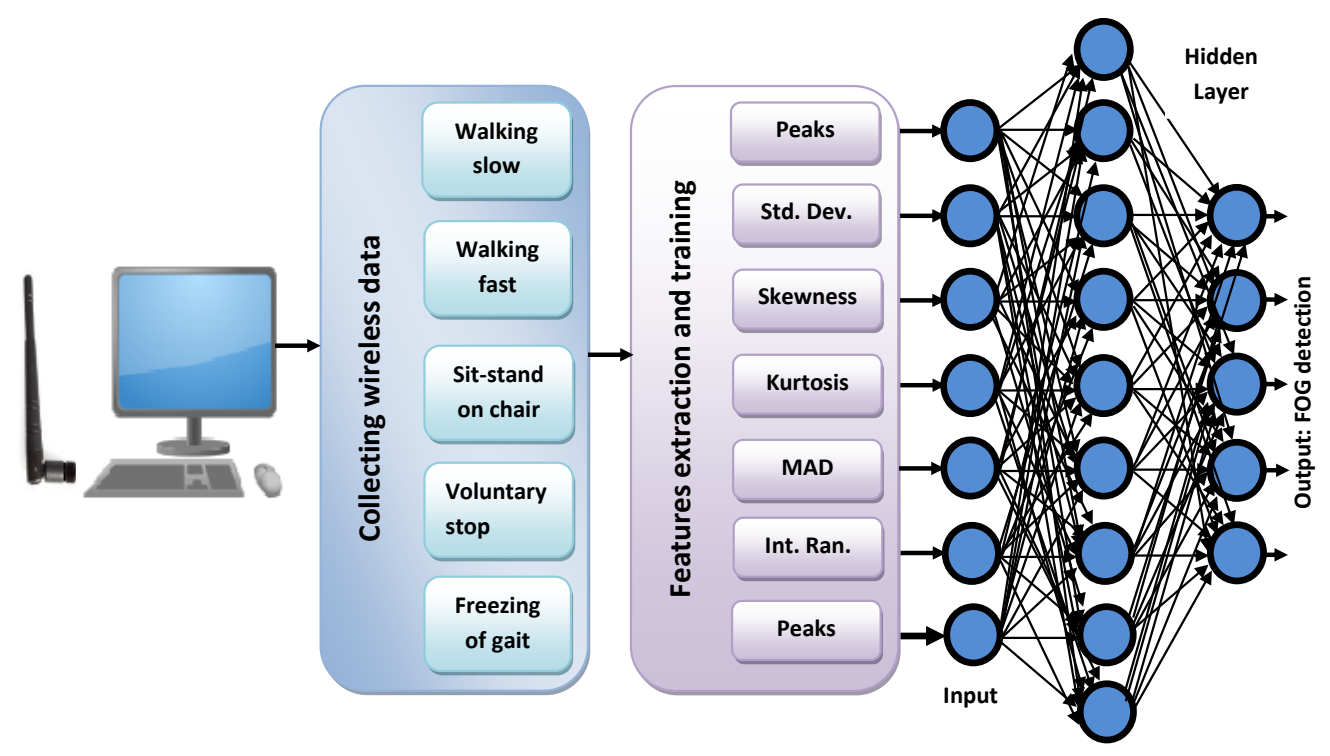

Fig. 2: Flowchart of the proposed FOG detection methodology.

Here $\mathbf{X}$ and $\mathbf{Y}$ are the transmitted and received signals, respectively. $\mathbf{N}$ denotes the channel noise while $\mathbf{H}$ demonstrates channel frequency response (CFR) of the wireless channel data which is a complex number.

$$
\begin{gathered}
\mathbf{H}=\left[\mathbf{h}_{\mathbf{1}}, \mathbf{h}_{\mathbf{2}}, \mathbf{h}_{\mathbf{3}}, \ldots \ldots ., \mathbf{h}_{\mathbf{n}}\right] \\
\mathbf{h}_{\mathbf{n}}=\left\|h_{n}\right\| \exp ^{j \angle h_{n}}
\end{gathered}
$$

In equation $3,\left\|h_{n}\right\|$ represents the variance of amplitude information and $\angle h_{n}$ describes phase information for $n$ sub-carrier. It should be noted that the phase information obtained via NIC is extremely random and cannot be used for any application. Therefore, in this paper we have used the variances of amplitude information training and testing the ANN algorithm to classify FOG from other daily life activities in an accurate and efficient way.

Step 2: In this step, time domain features such mean, standard deviation, skewness, kurtosis, mean absolute deviation (MAD), interquartile range (IQR) and peaks are extracted from the WCI data and plugged into the levenbergmarquardt (LM) training algorithm. Features extraction is primarily data reduction by finding the most informative variables-based subset of the same dataset.

Mean is defined as the average of all data points in a data matrix and specify the variability around a distinct value in some data matrix. Mean can be more effective in case of relatively uniformly spread data with no extraordinarily high 


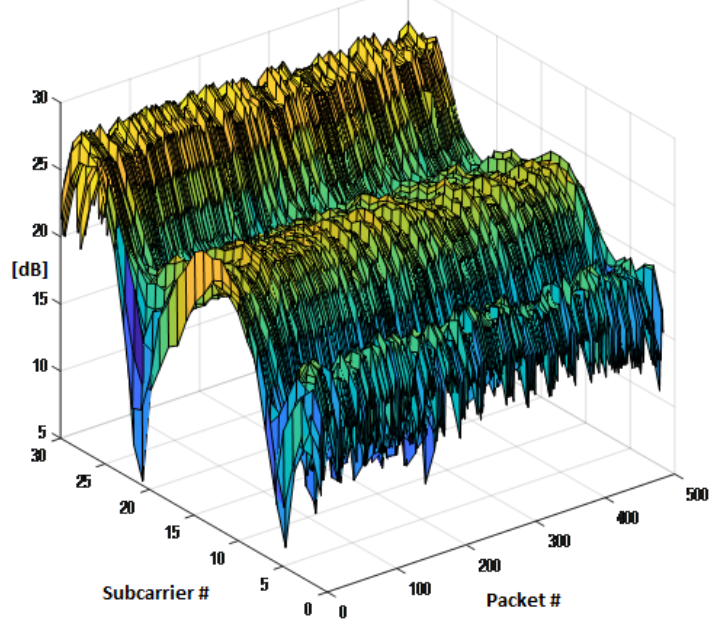

(a)

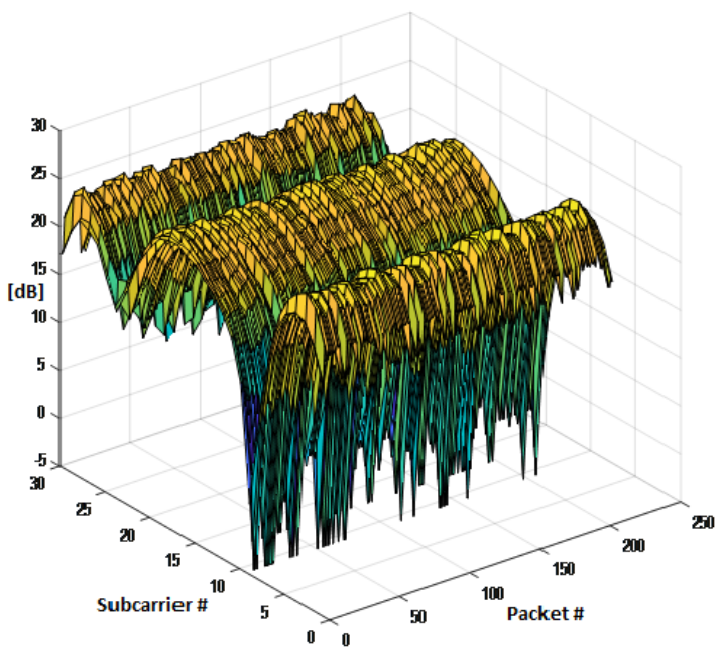

(c)

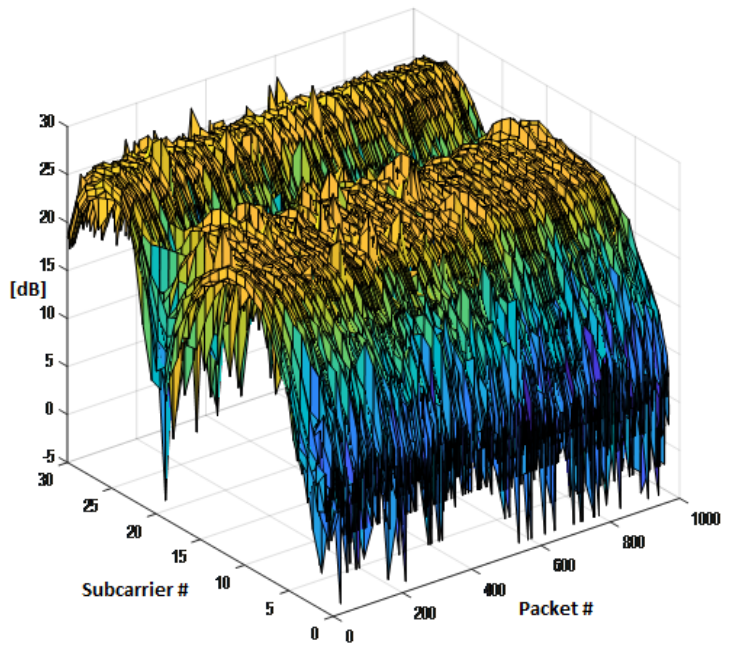

(b)

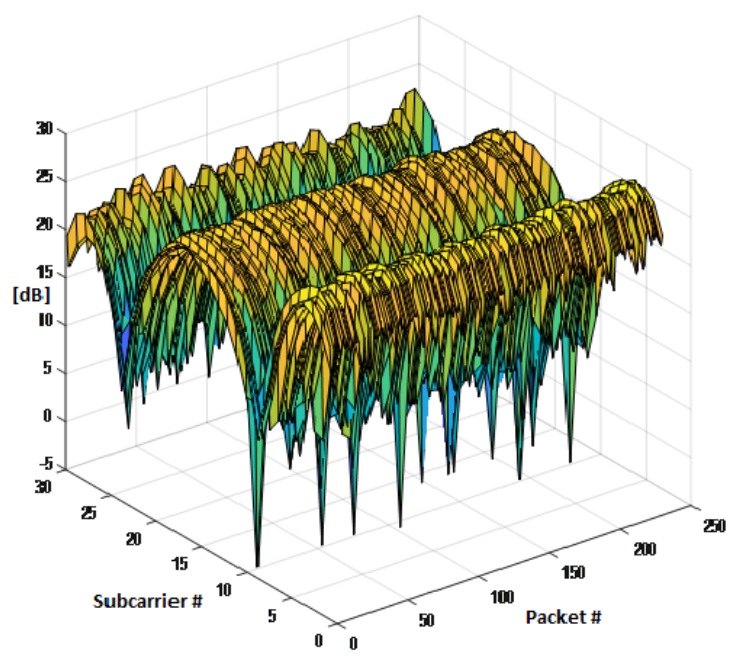

(d)

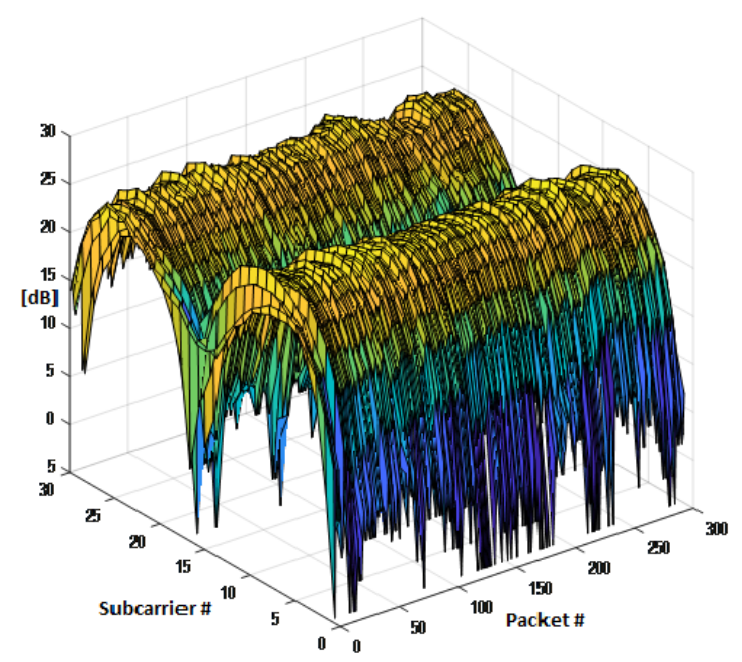

(e)

Fig. 3: Perturbations of amplitude information of 30 subcarriers. (a) Walking slow. (b) Walking fast. (c) Sit-stand on chair. (d) Voluntary stop. (e) FOG. 
or low values. Mathematically, mean is defined as:

$$
\mu_{x}=\frac{\sum_{i=1}^{a \times b} x_{i}}{a \times b},
$$

where $a$ and $b$ represent the number of rows and columns data matrix, respectively. $x_{i}$ is a data point at index $i$. Standard deviation is known as the spread (variability) of data points in a data matrix. Mathematically standard deviation $s d_{X}$ can be measured as [26]:

$$
s d_{x}=\frac{1}{a \times b} \sum_{i=1}^{a \times b}\left(x_{i}-\mu_{x}\right)^{2}
$$

Information about the spread of data can also be obtained via interquartile range [26]. $q_{u}$ computes the middle value above the median, while $q_{l}$ computes the middle value below the median of a data set. Interquartile range is written as:

$$
i q r=q_{u}-q_{l}
$$

Skewness $s_{x}$ computes the asymmetry of the probability distribution while kurtosis $k_{x}$ computes the shape of the probability distribution of a real-valued random variable. Skewness $s_{x}$ and kurtosis $k_{x}$ can be used to make judgments about image surfaces. Mathematically skewness and kurtosis can be computed as [27]:

$$
\begin{aligned}
& s_{x}=\frac{1}{a \times b} \times \sum_{i=1}^{a \times b}\left(\frac{x_{i}-\mu_{x}}{s d_{x}}\right)^{3} \\
& k_{x}=\frac{1}{a \times b} \times \sum_{i=1}^{a \times b}\left(\frac{x_{i}-\mu_{x}}{s d_{x}}\right)^{4}
\end{aligned}
$$

The mean absolute deviation about mean measure the dispersion of $\mathrm{X}$ about its mean and can be mathematically written as [28]:

$$
\operatorname{mad}_{x}=\frac{\sum_{i=1}^{a \times b}\left|x_{i}-\mu_{x}\right|}{a \times b}
$$

Step 3: Due to the faster operations, smaller training datasets requirement, easy implementation and ability to learn quickly, we have utilized a multi-layer perceptron neural network (MLPNN) with a single input layer, single hidden and single output layer as shown in Fig. 4. Levenberg-Marquardt (LM) [29] training algorithm is used during feature training process. LM is an iterative method that is used for solving non-linear minimization problem. The proposed classifier identify FOG episodes which is distinguishable from other routine activities using the proposed method. The input layer consists of seven neurons while the output layer consists of five neurons since we are classifying five different activities. Sigmoid activation function is used for input and output layers. Hidden layer which consist of ten neurons uses linear softmax activation function. Sigmoid 


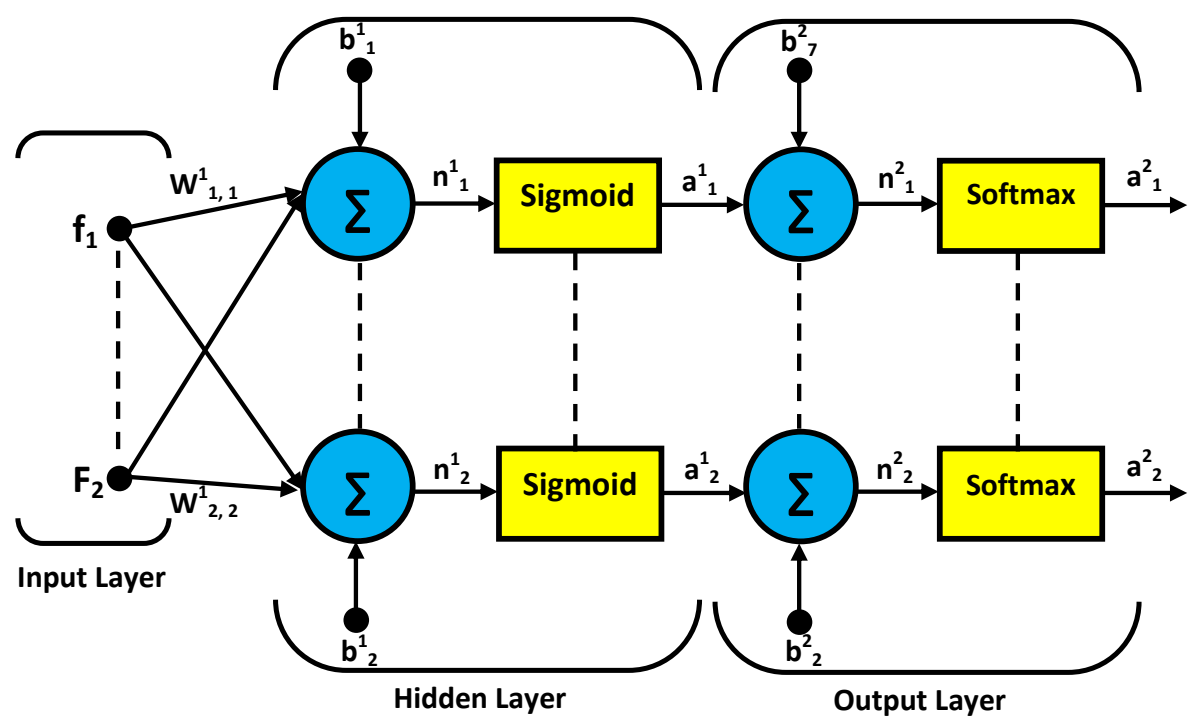

Fig. 4: MLPNN schematic diagram.

function maps the interval $(-\infty, \infty)$ onto $(0,1)$ while softmax function squashes an $x$ size vector between 0 and 1. Furthermore, softmax function normalized the exponential function to make the sum of whole vector equal to 1 . Therefore, the output softmax function interpret a set of specific features belong to certain class. Mathematically sigmoid $(\phi)$ and softmax $(\Phi)$ functions can be computed as in [30] and in [31], respectively:

$$
\begin{gathered}
\phi(x)=\frac{1}{1+\exp ^{-x}} \\
\Phi\left(x_{i}\right)=\frac{\exp ^{x_{i}}}{\sum_{n}^{N} \exp ^{x_{n}}}
\end{gathered}
$$

\section{Result and Discussion}

The variances of amplitude information for 30 subcarriers obtained using wireless devices exploiting $5 \mathrm{G}$ spectrum of five different activities is presented in Fig. 3, respectively. In Fig. 3, x-axis indicate total number of subcarriers, y-axis shows the total number of packets and z-axis is the relative power in $\mathrm{dB}$ indicating the variations in amplitude information. It can be observed that each human activity has resulted in a unique WCI signature which can be classified using multiclass ML-FFNN with the LM learning algorithm. Figure 5 shows the overall 




Fig. 5: Amplitude variation of a random subcarrier.

time history of five human activities for subcarrier number $6^{\text {th }}$. It illustrates the relative power level fluctuated between $4 \mathrm{~dB}$ to $16 \mathrm{~dB}$ for packet number 1 to 220. However, there is a shift in power level when the subject stands stationary (with small scale body movements such as breathing or small limb movements). Moreover, the power level varied around $24 \mathrm{~dB}$ when person was asked to walk slowly within area of interest. An increase in the variances of power level in packet numbers 900 to 1800 is observed when the person walks at a fast pace. While, for FOG episodes, the variations power level fluctuations between $24 \mathrm{~dB}$ to $26 \mathrm{~dB}$ are observed.

Table 1 illustrates the performance of our system as compared to the stateof-the-art latest works [7-11,32-36] in the domain of FOG detection leveraging traditional systems, such as wearable devices, smart phone sensors and vision based systems. The proposed system exploits $5 \mathrm{G}$ spectrum to detect and classify FOG with a high accuracy of $99.3 \%$ (see confusion matrix, Table 2) with an increase of approximately $6 \%$ over the second best method [33].

\section{Conclusion}

This study presented the design and implementation of an FOG system leveraging wireless devices operating at $4.8 \mathrm{GHz}$ (compatible with $5 \mathrm{G}$ spectrum for IoTs) in conjunction with multi-class softmax feedforward neural networks. The wireless channel information was extracted for five different human activities in indoor settings to classify the FOG episodes from sitting on chair, walking slowly, walking with fact pace and voluntary stop. The multi-class ML-FFNN leveraging features such as mean, standard deviation, skewness, kurtosis and peaks of power spectrum were used to classify the particular human activities. It was 
Table 1: Comparison of FOG detection systems

\begin{tabular}{|c|c|c|c|c|}
\hline Authors & Detection system & Types of sensors & Algorithm & Accuracy \\
\hline Prateek et al. [7] & Wearable devices & Inertial measurement unit & Generalized likelihood ratio test (GLRT) & $81.03 \%$ \\
\hline Camps et al. [8] & Wearable devices & Inertial measurement unit & Convolution neural network (CNN) & $89 \%$ \\
\hline Samà et al. [9] & Wearable devices & Accelerometer & Support vector machine & $89.6 \%$ \\
\hline Rodríguez et al. [32] & Wearable devices & Accelerometer & Support vector machine & $76.8 \%$ \\
\hline Aminis et al. [11] & Vision based & Camera, depth sensor & position/head offset \& angle tracking & $86.6 \% *$ \\
\hline Bigy et al. [10] & Vision based & Camera, depth sensor & subject/body joint positions & $92 \%$ \\
\hline Kim et al. [33] & Smart phone & Accelerometer, gyroscope & Convolution neural network (CNN) & $93.8 \%$ \\
\hline Capecci et al. [34] & Smart phone & Accelerometer & Power spectrum and cadence measures & $92.86 \%$ \\
\hline Kim et al. [35] & Smart phone & Accelerometer, gyroscope & AdaBoost.M1 & $86 \%$ \\
\hline Pepa et al. [36] & Smart phone & Accelerometer & Fuzzy inference system & $89 \%$ \\
\hline Proposed & 5G Spectrum & Wireless sensing & Multi-class softmax FFNN & $99.3 \%$ \\
\hline
\end{tabular}


Table 2: Confusion Matrix

\begin{tabular}{|c|c|c|c|c|c|c|}
\hline 1 & $\begin{array}{c}231 \\
10.5 \%\end{array}$ & $\begin{array}{c}0 \\
0.0 \%\end{array}$ & $\begin{array}{c}0 \\
0.0 \%\end{array}$ & $\begin{array}{c}1 \\
0.0 \%\end{array}$ & $\begin{array}{c}0 \\
0.0 \%\end{array}$ & $\begin{array}{c}99.6 \% \\
0.4 \%\end{array}$ \\
\hline 2 & $\begin{array}{c}2 \\
0.1 \%\end{array}$ & $\begin{array}{c}285 \\
13.0 \%\end{array}$ & $\begin{array}{c}0 \\
0.0 \%\end{array}$ & $\begin{array}{c}3 \\
0.1 \%\end{array}$ & $\begin{array}{c}0 \\
0.0 \%\end{array}$ & $\begin{array}{c}98.3 \% \\
1.7 \%\end{array}$ \\
\hline 3 & $\begin{array}{c}2 \\
0.1 \%\end{array}$ & $\begin{array}{c}0 \\
0.0 \%\end{array}$ & $\begin{array}{c}200 \\
9.1 \%\end{array}$ & $\begin{array}{c}1 \\
0.0 \%\end{array}$ & $\begin{array}{c}0 \\
0.0 \%\end{array}$ & $\begin{array}{c}98.5 \% \\
1.5 \%\end{array}$ \\
\hline 4 & $\begin{array}{c}0 \\
0.0 \%\end{array}$ & $\begin{array}{c}3 \\
0.1 \%\end{array}$ & $\begin{array}{c}0 \\
0.0 \%\end{array}$ & $\begin{array}{c}976 \\
44.4 \%\end{array}$ & $\begin{array}{c}1 \\
0.0 \%\end{array}$ & $\begin{array}{c}99.6 \% \\
0.4 \%\end{array}$ \\
\hline 5 & $\begin{array}{c}0 \\
0.0 \%\end{array}$ & $\begin{array}{c}0 \\
0.0 \%\end{array}$ & $\begin{array}{c}0 \\
0.0 \%\end{array}$ & $\begin{array}{c}3 \\
0.1 \%\end{array}$ & $\begin{array}{c}492 \\
22.4 \%\end{array}$ & $\begin{array}{c}99.4 \% \\
0.6 \%\end{array}$ \\
\hline & $\begin{array}{c}98.3 \% \\
1.7 \%\end{array}$ & $\begin{array}{c}99.0 \% \\
1.0 \%\end{array}$ & $\begin{array}{l}100 \% \\
0.0 \%\end{array}$ & $\begin{array}{c}99.2 \% \\
0.8 \%\end{array}$ & $\begin{array}{c}99.8 \% \\
0.2 \%\end{array}$ & $\begin{array}{c}99.3 \% \\
0.7 \%\end{array}$ \\
\hline
\end{tabular}

$\begin{array}{lllll}1 & 2 & 3 & 4 & 5\end{array}$

Target Class

observed that the system provided an average accuracy of $99.3 \%$ for various subjects under test.

\section{References}

1. J. Parkinson, "An essay on the shaking palsy (printed by whittingham and rowland for sherwood, neely, and jones)," 1817.

2. B. R. Bloem, J. M. Hausdorff, J. E. Visser, and N. Giladi, "Falls and freezing of gait in parkinson's disease: a review of two interconnected, episodic phenomena," Movement disorders: official journal of the Movement Disorder Society, vol. 19, no. 8, pp. 871-884, 2004.

3. C. G. Canning, S. S. Paul, and A. Nieuwboer, "Prevention of falls in parkinson's disease: a review of fall risk factors and the role of physical interventions," Neurodegenerative disease management, vol. 4, no. 3, pp. 203-221, 2014.

4. M. Amboni, P. Barone, M. Picillo, A. Cozzolino, K. Longo, R. Erro, and A. Iavarone, "A two-year follow-up study of executive dysfunctions in parkinsonian patients with freezing of gait at on-state," Movement Disorders, vol. 25, no. 6, pp. 800-802, 2010.

5. M. Zibetti, S. Angrisano, F. Dematteis, C. A. Artusi, A. Romagnolo, A. Merola, and L. Lopiano, "Effects of intestinal levodopa infusion on freezing of gait in parkinson disease," Journal of the neurological sciences, vol. 385, pp. 105-108, 2018.

6. M. Dagan, T. Herman, R. Harrison, J. Zhou, N. Giladi, G. Ruffini, B. Manor, and J. M. Hausdorff, "Multitarget transcranial direct current stimulation for freezing of gait in parkinson's disease," Movement Disorders, vol. 33, no. 4, pp. 642-646, 2018. 
7. G. V. Prateek, I. Skog, M. E. McNeely, R. P. Duncan, G. M. Earhart, and A. Nehorai, "Modeling, Detecting, and Tracking Freezing of Gait in Parkinson Disease Using Inertial Sensors," IEEE Transactions on Biomedical Engineering, vol. 65, no. 10, pp. 2152-2161, 2018.

8. J. Camps, A. Samà, M. Martín, D. Rodríguez-Martín, C. Pérez-López, J. M. Moreno Arostegui, J. Cabestany, A. Català, S. Alcaine, B. Mestre, A. Prats, M. C. Crespo-Maraver, T. J. Counihan, P. Browne, L. R. Quinlan, G. Laighin, D. Sweeney, H. Lewy, G. Vainstein, A. Costa, R. Annicchiarico, À. Bayés, and A. Rodríguez-Molinero, "Deep learning for freezing of gait detection in Parkinson's disease patients in their homes using a waist-worn inertial measurement unit," Knowledge-Based Systems, vol. 139, pp. 119-131, 2018.

9. A. Samà, D. Rodr \'\iguez-Mart \'\in, C. Pérez-López, A. Català, S. Alcaine, B. Mestre, A. Prats, M. C. Crespo, and À. Bayés, "Determining the optimal features in freezing of gait detection through a single waist accelerometer in home environments," Pattern Recognition Letters, vol. 105, pp. 135-143, 2018.

10. A. A. M. Bigy, K. Banitsas, A. Badii, and J. Cosmas, "Recognition of postures and freezing of gait in parkinson's disease patients using microsoft kinect sensor," in Neural Engineering (NER), 2015 7th International IEEE/EMBS Conference on. IEEE, 2015, pp. 731-734.

11. A. Amini, K. Banitsas, and W. R. Young, "Kinect4fog: monitoring and improving mobility in people with parkinson?s using a novel system incorporating the microsoft kinect v2," Disability and Rehabilitation: Assistive Technology, pp. 1-8, 2018.

12. M. G. Rahim, C. C. Goodyear, W. B. Kleijn, J. Schroeter, and M. M. Sondhi, "On the use of neural networks in articulatory speech synthesis," The Journal of the Acoustical Society of America, vol. 93, no. 2, pp. 1109-1121, 1993.

13. S.-Y. Kung and J.-S. Taur, "Decision-based neural networks with signal/image classification applications," IEEE Transactions on Neural Networks, vol. 6, no. 1, pp. 170-181, 1995.

14. W. Chu and N. Bose, "Speech signal prediction using feedforward neural network," Electronics Letters, vol. 34, no. 10, pp. 999-1001, 1998.

15. D. DeKruger and B. R. Hunt, "Image processing and neural networks for recognition of cartographic area features," Pattern Recognition, vol. 27, no. 4, pp. 461-483, 1994.

16. E. Cosatto and H. P. Graf, "A neural network accelerator for image analysis," IEEE Micro, no. 3, pp. 32-38, 1995.

17. V. Kvasnička, "An application of neural networks in chemistry," Chemical Papers, vol. 44, no. 6, pp. 775-792, 1990.

18. B. Lerner, M. Levinstein, B. Rosenberg, H. Guterman, L. Dinstein, and Y. Romem, "Feature selection and chromosome classification using a multilayer perceptron neural network," in Neural Networks, 1994. IEEE World Congress on Computational Intelligence., 1994 IEEE International Conference on, vol. 6. IEEE, 1994, pp. 3540-3545.

19. S. Lek and J.-F. Guégan, "Artificial neural networks as a tool in ecological modelling, an introduction," Ecological modelling, vol. 120, no. 2-3, pp. 65-73, 1999.

20. R. Colasanti, "Discussions of the possible use of neural network algorithms in ecological modeling." Binary: Computing in Microbiology, vol. 3, no. 1, pp. 13-15, 1991.

21. X. Yang, S. A. Shah, A. Ren, N. Zhao, J. Zhao, F. Hu, Z. Zhang, W. Zhao, M. U. Rehman, and A. Alomainy, "Monitoring of patients suffering from rem sleep behav- 
ior disorder," IEEE Journal of Electromagnetics, RF and Microwaves in Medicine and Biology, vol. 2, no. 2, pp. 138-143, 2018.

22. X. Yang, S. A. Shah, A. Ren, N. Zhao, D. Fan, F. Hu, M. Ur-Rehman, K. M. von Deneen, and J. Tian, "Wandering pattern sensing at s-band," IEEE Journal of Biomedical and Health Informatics, 2017.

23. X. Wang, C. Yang, and S. Mao, "Tensorbeat: Tensor decomposition for monitoring multiperson breathing beats with commodity wifi," ACM Transactions on Intelligent Systems and Technology (TIST), vol. 9, no. 1, p. 8, 2017.

24. D. Zhang, H. Wang, and D. Wu, "Toward centimeter-scale human activity sensing with wi-fi signals," Computer, vol. 50, no. 1, pp. 48-57, 2017.

25. W. Cao, X. Li, W. Hu, J. Lei, and W. Zhang, "Ofdm reference signal reconstruction exploiting subcarrier-grouping-based multi-level lloyd-max algorithm in passive radar systems," IET Radar, Sonar \& Navigation, vol. 11, no. 5, pp. 873-879, 2016.

26. E. Kreyszig, Advanced engineering mathematics. John Wiley \& Sons, 2010.

27. V. Kumar and P. Gupta, "Importance of statistical measures in digital image processing," International Journal of Emerging Technology and Advanced Engineering, vol. 2, no. 8, pp. 56-62, 2012.

28. E. A. H. El Amir, "On uses of mean absolute deviation: decomposition, skewness and correlation coefficients," Metron, vol. 70, no. 2-3, pp. 145-164, 2012.

29. M. T. Hagan, H. B. Demuth, M. H. Beale, and O. De Jesús, Neural network design. Pws Pub. Boston, 1996, vol. 20.

30. B. Karlik and A. V. Olgac, "Performance analysis of various activation functions in generalized mlp architectures of neural networks," International Journal of Artificial Intelligence and Expert Systems, vol. 1, no. 4, pp. 111-122, 2011.

31. Y. Tang, "Deep learning using linear support vector machines," arXiv preprint arXiv:1306.0239, 2013.

32. D. Rodríguez-Martín, A. Samà, C. Pérez-López, A. Català, J. M. Moreno Arostegui, J. Cabestany, À. Bayés, S. Alcaine, B. Mestre, A. Prats, M. C. Crespo, T. J. Counihan, P. Browne, L. R. Quinlan, G. ÓLaighin, D. Sweeney, H. Lewy, J. Azuri, G. Vainstein, R. Annicchiarico, A. Costa, and A. Rodríguez-Molinero, "Home detection of freezing of gait using support vector machines through a single waist-worn triaxial accelerometer," Plos One, vol. 12, no. 2, p. e0171764, 2017. [Online]. Available: http://dx.plos.org/10.1371/journal.pone.0171764

33. H. B. Kim, H. J. Lee, W. W. Lee, S. K. Kim, H. S. Jeon, H. Y. Park, C. W. Shin, W. J. Yi, B. Jeon, and K. S. Park, "Validation of Freezing-of-Gait Monitoring Using Smartphone," Telemedicine and e-Health, vol. 24, no. 12, p. tmj.2017.0215, 2018. [Online]. Available: https://www.liebertpub.com/doi/10.1089/tmj.2017.0215

34. M. Capecci, L. Pepa, F. Verdini, and M. G. Ceravolo, "A smartphonebased architecture to detect and quantify freezing of gait in Parkinson's disease," Gait and Posture, vol. 50, pp. 28-33, 2016. [Online]. Available: http://dx.doi.org/10.1016/j.gaitpost.2016.08.018

35. H. Kim, H. J. Lee, W. Lee, S. Kwon, S. K. Kim, H. S. Jeon, H. Park, C. W. Shin, W. J. Yi, B. S. Jeon, and K. S. Park, "Unconstrained detection of freezing of Gait in Parkinson's disease patients using smartphone," Proceedings of the Annual International Conference of the IEEE Engineering in Medicine and Biology Society, $E M B S$, vol. 2015-November, pp. 3751-3754, 2015.

36. L. Pepa, L. Ciabattoni, F. Verdini, M. Capecci, and M. G. Ceravolo, "Smartphone based Fuzzy Logic freezing of gait detection in Parkinson's Disease," MESA 2014 - 10th IEEE/ASME International Conference on Mechatronic and Embedded Systems and Applications, Conference Proceedings, 2014. 